\title{
Reasoning about Semantic Web in Isabelle/HOL
}

\author{
Yue Tang \\ School of Computing \\ National University of Singapore \\ Republic of Singapore \\ tangy@comp.nus.edu.sg \\ Jin Song Dong \\ School of Computing \\ National University of Singapore \\ Republic of Singapore \\ dongjs@comp.nus.edu.sg
}

\author{
Jing Sun \\ Department of Computer Science \\ The University of Auckland \\ New Zealand \\ j.sun@cs.auckland.ac.nz \\ Brendan Mahony \\ Information Technology Division \\ Defence Science and Technology \\ Organization of Australia \\ Brendan.Mahony@dsto.defence.gov.au
}

\begin{abstract}
Semantic Web is regarded as the next generation of the World Wide Web. It provides not only the structure of the web but also meaningful semantics for the information presented. To make Semantic Web services understandable for distributed agents, formal definitions of the ontologies and their consistencies are essential. However, the existing tools for reasoning about Semantic Web ontologies are still primitive. We believe that mature Software Engineering tools, such as theorem provers, can contribute to the reasoning phase. In this paper, we present an approach of encoding the Semantic Web ontology (DAML+OIL) into the generic theorem prover Isabelle/HOL for automatic reasoning. Furthermore, a translation tool was developed to transform Semantic Web ontologies into their extended Isabelle theories. With additional intermediate lemmas, Isabelle can be used to perform both subsumption (class) level and instantiation (instance) level reasoning of the Semantic Web ontologies.

Keywords: Semantic Web, DAML+OIL, Theorem proving, Isabelle/HOL.
\end{abstract}

\section{Introduction}

Recent research on the World Wide Web have extended to the semantics of the web content. More meaningful information is embedded into the web content, which makes it possible for intelligent agent programs to retrieve related information based on their requirements. The Semantic Web [2] approach proposed by the World Wide Web Consortium (W3C) attracts the most attention. It is regarded as the next generation of the web. A Semantic Web service is a web application developed based on the Semantic Web technology. There have been some Semantic Web services developed recently, e.g., ITTALKS [4]. Ontology is one of the important concepts in Semantic Web services. An ontology is a document defining the semantic relationships between terms used in a Web service. A Semantic Web service usually works in a distributed environment due to the nature of the web. Hence, the consistency of its ontology is essential to make the service work correctly. Because the Semantic Web technology is still a relatively new research field, it is lacking in supporting tools for reasoning about ontology consistencies. Recently, some effects have been put to meet this needs. OilEd [1] is a graphical ontology editor. Fast Classification of Terminologies (FaCT) [9] is a Description Logic (DL) classifier. FaCT is built in OilEd as a reasoner. The FaCT system works on Description Logic, which is the basis of the Semantic Web. When it is invoked, FaCT can point out possible inconsistencies and help OilEd to show the graphical class hierarchy. However, FaCT has a main restriction. It can only support conceptual level reasoning but not instance level reasoning. Hence, it is impossible for FaCT to check instantiation relations. Renamed ABox and Concept Expression Reasoner (RACER) [8] is a TBox and ABox reasoner for description logic SHIQ [10]. Similar to FaCT, RACER is implemented in Common Lisp. It is a client-server system. The front-end is called RACER Interactive Client Environment (RICE). They are connected through the socket-based TCP/IP interface. RACER has been applied to projects in Semantic Web and software engineering. It has an advantage over FaCT that it can support both conceptual level and instance level reasoning. Open World Assumption (OWA) is 
adopted by RACER. It means that 'what cannot be proven to be true is not believed to be false'. RACER returns true as long as it cannot deduce the result is false. This may cause incorrectness of results that RACER returns.

At the same time, there are many existing tools that can support logical reasoning very well in the software engineering domain. It is believed that the Semantic Web could become an novel application domain for software modeling. The consequent research is to encode Semantic Web ontology into a formal modeling language, then use an existing proof tool to perform automated reasoning. Recently, there are some approaches of using formal modeling tools for ontology consistency checking. Alloy is a light-weight software modeling language based on first order logic. The approach of using Alloy to check and reason about Semantic Web [6] makes it possible to support an automated ontology verification. A Semantic Web ontology is transformed from its DAML+OIL representation into an Alloy specification. Alloy Analyzer then analyzes the model to check the consistency of the ontology. This approach can support both conceptual level and instance level reasoning. But it has its own limitations. A finite scope must be provided for AA to perform analysis. Most of the time this is not a problem as long as the scope is small. However, if the Semantic Web ontology is widely distributed through the Internet, the scope could be hard to define. Thus the confidence in the result will depend on the size of the scope adopted. $\mathrm{Z}$ is a formal specification language based on set theory and predicate logic. Z/EVES [12] is a theorem proving tool for the $\mathrm{Z}$ language. One of the approaches is checking the ontology consistency using $\mathrm{Z}$ with its proof tool Z/EVES [5]. Unlike the approach of Alloy, it does not have a limitation on the scope. This is because Z/EVES is a theorem prover and can support large scale proofs. Hence, it provide us with confidence in the consistency of an ontology. Unfortunately it is not yet a perfect approach. Like Alloy, Z/EVES depends too much on the underlying formal modeling language Z. Semantic Web ontology has to be converted into Z/Alloy models in order to perform consistency verifications. The process of such translation may cause inconsistency as well.

In this paper, we present an approach of directly encoding the Semantic Web meta-language (DAML+OIL) into the generic theorem prover Isabelle [11] for consistency reasoning. There are three main contributions of this paper, i.e., an formal definition of the DAML+OIL semantics in the Isabelle high order logic, a transformation program for translating Semantic Web ontology into their Isabelle theories and an automatic ontology reasoning environment provided by the Isabelle theorem prover. Firstly, an Isabelle theory library is defined for the generic DAML+OIL semantics. With this theory, concrete examples of Semantic Web ontologies are expressed as its extensions. Consequently, a transformation program for translating an ontology into its corresponding Isabelle theory becomes necessary. This program is developed based on an existing Java library for DAML+OIL. It is reusable and extensible for future enhancement. Finally, based on the Isabelle proving facilities, we can perform automatic ontology consistency checking both at conceptual and instance levels.

The remainder of the paper is organized as follows. Section 2 briefly introduces the background knowledge for Semantic Web and the generic theorem prover Isabelle. Section 3 presents Isabelle theory definitions for DAML+OIL semantics. Section 4 introduces a program that can transform a Semantic Web ontology into its Isabelle theory representation. Section 5 demonstrates a case study on a complete ontology reasoning process: starting from transformation, followed by goal identifications, ending with additional supporting lemmas and final proofs. Examples of both class-level reasoning and instance-level reasoning are presented. section 6 concludes the paper and discusses about future work.

\section{Backgrounds}

\subsection{Semantic Web overview}

The Semantic Web [2] has been considered as the next generation of the World Wide Web. It not only includes structural and visible data like traditional WWW, but also describes data with machine-understandable semantics. In a word, web contents become readable to intelligent software agents in addition to human beings.

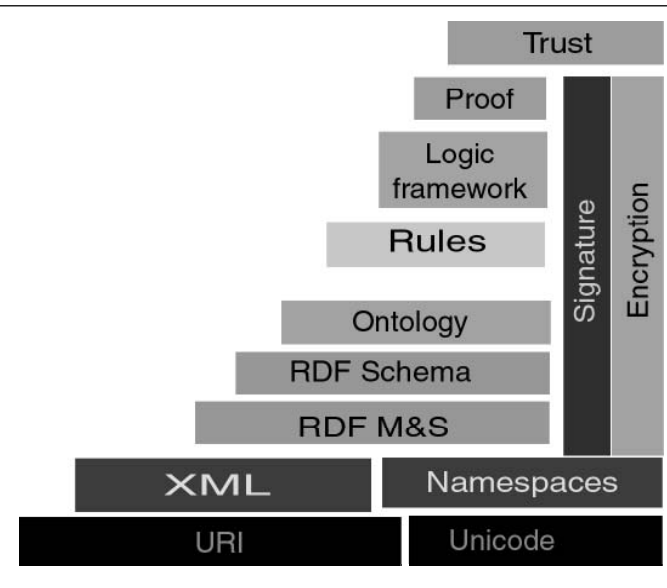

Figure 1. Semantic Web layers proposed by Tim Berners-Lee 
Figure 1 shows the structure of the Semantic Web layers, a vision from Tim Berners-Lee, the creator of the WWW. It illustrates a clear picture of relations between all layers. We put our focus on the ontology (DAML+OIL) related layers, i.e., from the RDF and RDFS up to the logic framework and proof layers (in Isabelle).

\subsubsection{RDF and RDFS}

In order to fulfill the requirements of understandable data in the Semantic Web, ontologies are defined and distributed throughout the World Wide Web. An ontology can be viewed as a kind of XML document containing the formal definitions of terminologies and their relations. The Resource Description Framework (RDF) [7] forms a foundation for intelligent agents to exchange information. It provides a framework for meta-data processing. An RDF statement is a logic triple that has the form: <subject property object>, where subject refers to a Web resource, property and object describe one of its properties (relations) with other web resources. Hence, in contrast to a simple XML document, an RDF document is more semantically meaningful. The RDF Schema (RDFS) [7] provides a vocabulary for RDF documents in addition to their RDF model and syntax. In addition, RDFS also puts constraints on RDF structures. Therefore, it can help software agents to explicitly 'understand' (extract) the information in a Semantic Web document.

\subsubsection{DAML+OIL}

The DARPA Agent Markup Language (DAML) [7] is a web ontology language based on description logic. Combined with the Ontology Interchange Language (OIL) [3], DAML+OIL [13] is developed on the basis of XML and RDFS with well-defined semantics. It provides more logic constructs for defining classes and properties as well as instances, domains and ranges of properties. It is much more powerful than RDFS on modeling Semantic Web ontologies.

The following is an example ontology in DAML+OIL. It defines a web resource Person with relations and restrictions. Animal is a class and hasparent is a property. Person is a subclass of Animal and has the property hasparent.

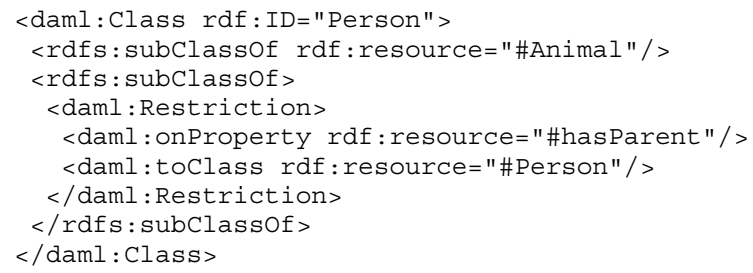

With the DAML+OIL language, constraints between different web resources can be specified to a deeper content. Thus logical relationships in the ontology can be made more machine understandable to their semantic web services.

\subsection{Isabelle overview}

Isabelle [11] is a generic proof system for implementing logical formalisms. It provides powerful mechanisms in defining hierarchical logic theories (object logics). New object logics can be built from Isabelle metalogic, by means of constructing and proving new theories. Its fundamental inference techniques are based on higher order unification and term rewriting.

\subsubsection{Isabelle theory library}

Isabelle supports library definitions. A library is called theory in Isabelle. It can be either pre-defined or user-defined. Isabelle is released with a wide range of pre-defined logic theories, such as First Order Logic (FOL), Higher Order Logic (HOL), Zermelo Frankel set theory (ZF), Constructive Type Theory (CTT), the Logic of Computable Functions (LCF), and so on. In addition, users can also define their own theories, e.g., MyTheory . thy. The main content of a theory file can include type declarations, function definitions, lemmas and proofs.

\subsubsection{Definitional modeling using Isabelle/HOL}

Isbelle/HOL [11] implements the Isabelle high order logic library. Some of the building blocks in developing Isabelle logics are as follows.

- Theory definition - New theories are defined as an extension on the existing theories. Thus definitions and lemmas and proof rules can be inherited. The general form of the theory definition command is:

$$
\begin{aligned}
& \text { theory MyTheory }=\text { theory }_{1}+\ldots+\text { theory }_{n} \\
& \text { : where theory } 1, \ldots \text {, theory } n \text { are exist- } \\
& \text { ing supporting theories. }
\end{aligned}
$$

- Type definition - New types are constructed by the declaration typedecl or datatype. Isabelle supports recursive datatype definitions. The general form of a type definition is:



- Function definitions - Functions are defined by their name, inputs, outputs and their actual definitions. They provide essential information for constructing proofs. A function can be defined as follows. 
consts myFunction : : "nat list $=>$ nat $=>$ bool" defs myFunction def : "myFunction $\mathrm{NL} V==$

ALL $n:($ set NL). $n=V "$

- Lemmas and Theorems - The goal to be proved in a theory is defined as lemma or theorem. It is a logic statement. Theorems and lemmas can be proved by applying proof rules and tactics. The format of lemma and theorem definitions are as follows.

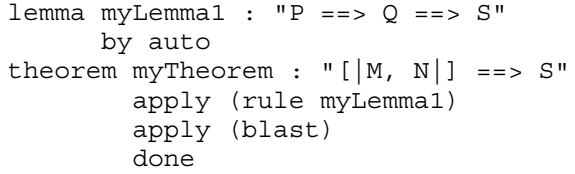

\section{Isabelle theory definition for DAML+OIL}

\subsection{Basic concepts}

DAML+OIL is based upon RDF and RDFS with the addition of class, property and object concepts. DAML+OIL primitives are encoded into Isabelle definitions according to their semantics [7].

An Isabelle theory SW is defined for the semantic model of DAML+OIL meta-language using HOL as the basic logic library.

theory SW = Main:

Based on the above sematic web DAML+OIL definition, new Semantic Web ontologies can be created as extensions to the SW theory. For example,

theory ontologyExample = SW:

Everything in the Semantic Web context is a type of resource. It can be classified as a class, a property or an instance resource type. To specify these types and the relationship between them, three axiomatic types are declared and one data type SWresource is created as follows.

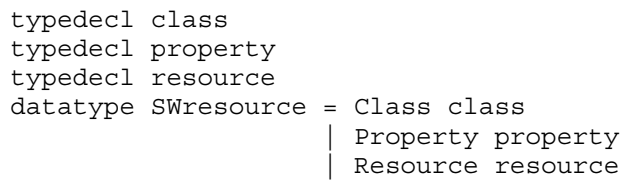

A class is a kind of resource that is related to a set of instances in some category. The instances can be any type of resource, i.e., SWresource. The function instanceof declares the relationship between a class and its set of instances which can be any type of Semantic Web resource. Given a class, this function returns a set of SWresource that represents the instances of the class.

consts instanceof : : "class => SWresource set"

A property also has a basic relation called subVal. It declares the relationship between a property and a set of subject-value pairs. Given a property, this function returns a set of SWresource pairs. consts subval : :

"property $=>$ (SWresource * SWresource) set"

\subsection{Class elements}

A class elements mainly describe the relations among classes in DAML+OIL. In this section, four commonly used relations are encoded into Isabelle, i.e., subClassof, disjointWith, sameClassAs and disjointUnionof.

\subsection{1. subClassOf}

The subclassof function describes the relation between two classes. A class $\mathrm{C} 1$ is subclass of another class $\mathrm{C} 2$ if and only if all C1's instances are also inside class $\mathrm{C} 2$. The declaration and definition of subClassOf are as follows.

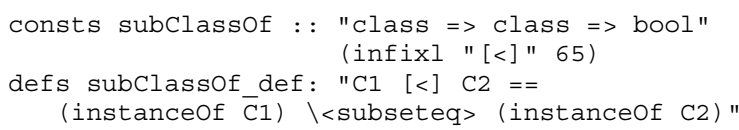

where the 'infixl' statement defines a binary symbol ' $[<]$ ' introduced for the subClassof relation.

\subsection{2. disjointWith}

The disjointWith function defines that two classes $\mathrm{C} 1$ and $\mathrm{C} 2$ are disjoint so that they have no common set of instances.

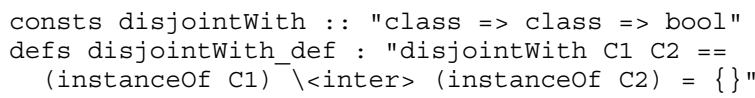

The sameClassAs and disjointUnionof functions are defined similarly.

\subsection{Property restrictions}

The property restrictions focus on the relationships between web resources. In this subsection, some commonly used property restrictions are discussed.

\subsection{1. toClass}

According to DAML+OIL semantics, the function toClass constructs a class $\mathrm{C} 1$ with a restriction on its instances. An instance of $\mathrm{C} 1$ either has no values in property $\mathrm{P}$, or if it has values in property $\mathrm{P}$, the respective values must belong to another class $\mathrm{C} 2$.

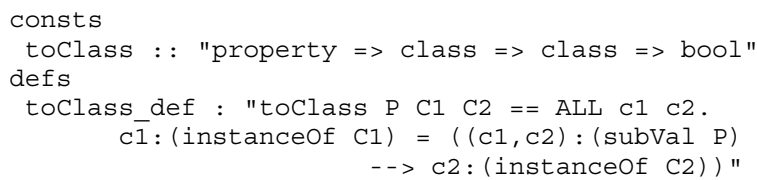




\subsection{2. hasValue}

The function hasValue restricts the property value for a class $C$. All the instances of class $C$ have the same value of property $\mathrm{P}$.

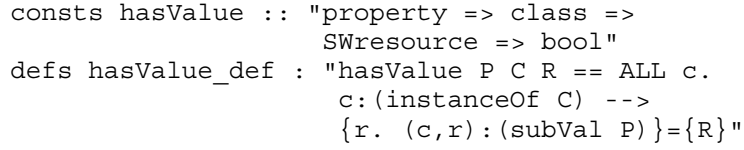

Other property restrictions such as hasClass, cardinality, maxCardinality, minCardinality, cardinality $Q$, maxCardinality $Q$, minCardinality $Q$ are defined correspondingly.

\subsection{Property elements}

\subsection{1. subPropertyOf}

The function subPropertof defines a relation between two properties. One property $\mathrm{P} 1$ is said to be a sub-property of another property P2 if and only if the set of subject-value pairs in $\mathrm{P} 1$ is a subset of that in $\mathrm{P} 2$.

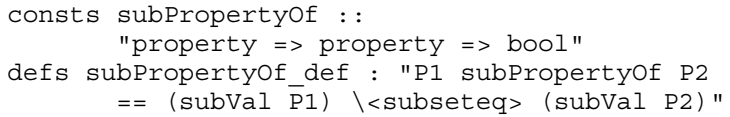

\subsection{2. domainOf}

The function domainof restricts the domain of a property $\mathrm{P}$. All subjects from $\mathrm{P}$ are instances of a domain class $\mathrm{C}$.

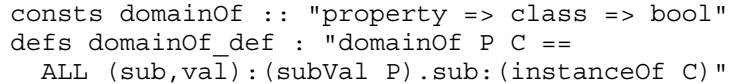

\subsection{3. transitiveProp}

The function transitiveProp defines the transitivity of the subject-value pairs in a property $\mathrm{P}$.

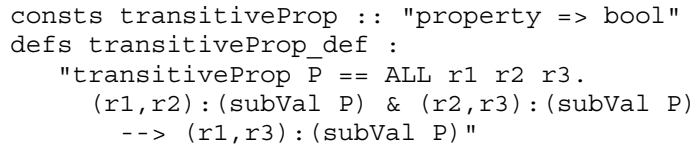

Other property elements such as rangeOf, samePropertyAs, inverseOf, uniqueProp, unambigousProp are defined in a similar way.

In this section, an Isabelle encoding (SW. thy) of the DAML+OIL semantic web language in Higher Order Logic (HOL) is presented. We have defined a complete library of the DAML+OIL semantics in Isabelle. Due to the space limitation of the paper, only parts of the encoding are listed here. This generic theory (SW.thy) acts as a foundation library for modeling other user-defined Semantic Web ontologies.

\section{Transform from DAML+OIL to Isabelle}

In section 3, we defined an Isabelle theory (SW.thy) for DAML+OIL semantics. The consequent task is to develop a tool to transform a Semantic Web ontology into its corresponding Isabelle theory file. Our DAML2Isa translation tool was implemented based on the Jena semantic web toolkit developed by the HP Labs Semantic Web Research Group.

\subsection{Transformation rules}

The DAML2Isa tool implements transformation rules from DAML+OIL ontology to Isabelle theory. Every Semantic Web ontology is transformed as a new theory file in Isabelle. The theory SW . thy acts as a base library for the new theories. An ontology information includes class, property, restriction, etc. Resources, such as classes, properties and general resources, are transformed as constant definitions in Isabelle, while restrictions are transformed as axioms. A brief description of the translation rules are as follows.

- Class transformation - A DAML+OIL class is transformed to an Isabelle constant definition with class type. The RDF ID is extracted as the class name. Axioms are generated based on the constraints that this class has.

- Property transformation - A property is transformed as a constant definition with property type. The RDF ID is taken as the property name. Properties may have property elements, which are transformed to Isabelle axioms.

- Restriction transformation - Property restrictions are transformed to axiom definitions in Isabelle. The axiom name is composed of the restriction name and property name followed by other related information.

Detailed translation rules are implemented in the DAML2ISa transformation tool. DAML+OIL ontologies can be automatically translated from their XML representations into their corresponding Isabelle theories. In the next section, we will demonstrate a complete consistency reasoning process of a 'Human Being' ontology case study using Isabelle.

\section{Reasoning about Semantic Web ontology}

\subsection{Reasoning procedure}

Figure 2 shows the proof procedure for reasoning about DAML+OIL Semantic Web ontology in the Isabelle theorem prover. First, an ontology in DAML+OIL is input into the DAML2Isa transformation program. The DAML2Isa 


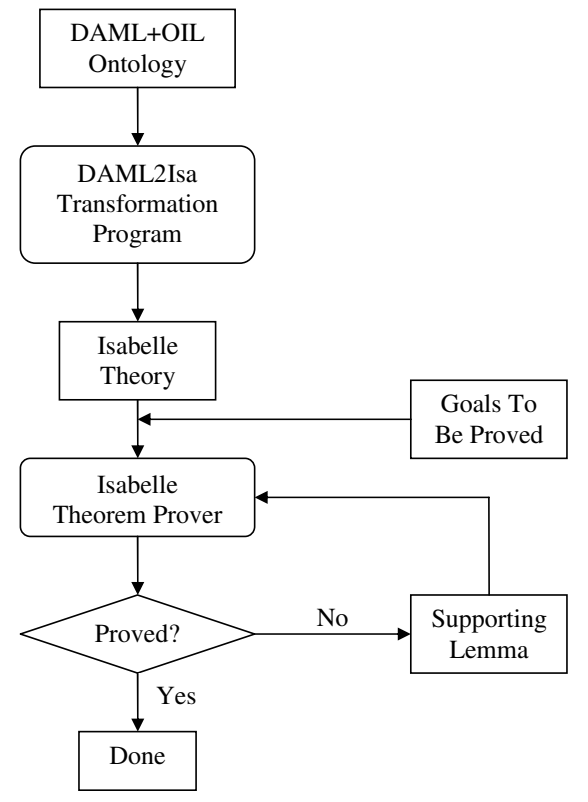

Figure 2. Ontology reasoning using Isabelle

produces an output file containing the generated Isabelle theory. After adding the goals to be proved, the file is input into Isabelle. If the goals can be proved instantly using Isabelle default tactics, it is done. Otherwise, it is necessary to add more supporting lemmas and pass the document to Isabelle again until all goals are proved.

\subsection{Example ontology}

A partial DAML+OIL example ontology about 'Human Beings' is used as a case study to illustrate the procedures of reasoning about Semantic Web ontology using Isabelle. This example ontology is available at the DAML web site for easy reference. The ontology defines four resource classes, Animal, Person, Male and Female, and their properties. The DAML+OIL ontology (in XML) can be translated into its corresponding Isabelle theory by the DAML2Isa tool automatically. After transforming a DAML+OIL ontology into its Isabelle theory, the next step is to identify the proof goals and apply reasoning tactics to perform the verification.

\subsection{Subsumption reasoning}

The purpose of subsumption reasoning is to verify the subclass relationship between two classes. This can be done through different ways of inference based on the information presented in the ontology.

\subsubsection{Transitivity of a subclass}

The most direct way of subsumption reasoning is based on the transitive property of a subclass relation. For example, suppose there is a class Man which is a subclass of Person and Male, we can infer that class Man is also a subclass of the Animal class. The goal that class Man is a subclass of Animal is defined in Isabelle as a theorem:

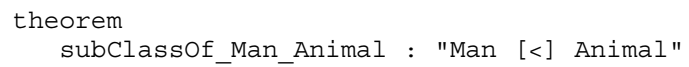

In order to prove this goal, a supporting lemma is needed in the main theory SW . thy to express the transitivity property of a subclassof function.

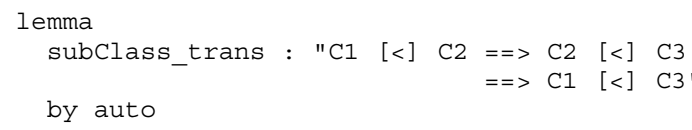

The above lemma can be easily proved using the Isabelle auto proof tactic. With this supporting lemma, our original goal theorem subClassOf_Man_Animal can be proved based on combining the other two axioms.

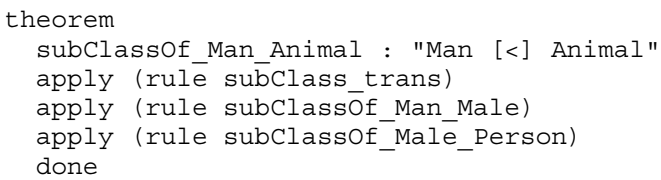

Note that the apply command denotes to apply a particular proof rule or tactic in attempts to solve a goal.

\subsubsection{Class restriction on property}

Another widely used method of subsumption reasoning is based on class restrictions on properties. If a class $\mathrm{C} 1$ has a restriction on property $\mathrm{P}$, and another class $\mathrm{C} 2$ has the same restriction on property $\mathrm{P}$ and possibly additional restrictions, then class $\mathrm{C} 2$ is a subclass of $\mathrm{C} 1$. For example, suppose a class Adult is subclass of Animal and has restriction toclass on property hasparent.

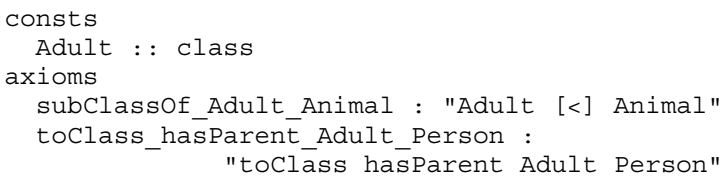

The following presents a supporting lemma for proving this goal based on functions subClassof and toClass.




Provided with the above additional lemma, the goal subClassOf_Adult_Person can be proved effectively as follows.

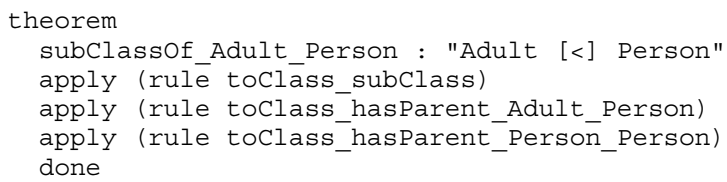

\subsection{Instantiation reasoning}

As stated previously, Isabelle can support instance level reasoning for Semantic Web ontologies. Instance level reasoning focuses on the relation between a Semantic Web resource and a class.

\subsubsection{Membership of a class}

A Semantic Web resource can be proved to be an instance of a class as long as there is enough information. For instance, based on set theory, if an element (Semantic Web resource) is an instance of a set (class), it must be an instance of any super set (super class). Here is a more complicated case. Suppose that there exist two Semantic Web resources anAdult and aPerson. The resource anAdult is an instance of class Animal, aperson is an instance of class Person, and the resource anAdult has a property hasParent on aPerson. We can prove that anAdult is also an instance of the Person class.Once again, a supporting lemma is necessary for the proof. The lemma toclassD1 explains more about the function toclass.

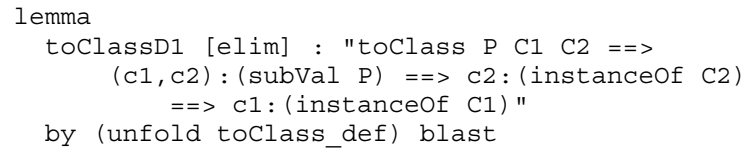

With the three additional axioms, the requirement for proving the theorem is fulfilled. It can be concluded that anAdult is an instance of the class Person.

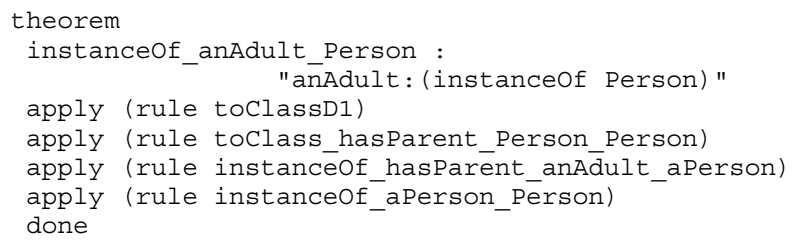

\subsubsection{Non-membership of a class}

Although Isabelle does not support model checking, users can still perform inconsistency checking at an instance level. For example, sometimes an inconsistency can be found by proving that a Semantic Web resource is not an instance of a certain class. As stated in the example ontology, class Female is disjoint with the class Male. Suppose there is a resource aFemale which is an instance of Female. It is obvious that aFemale is not an instance of Male. In order to prove this, a supporting lemma disjointWithD is necessary.

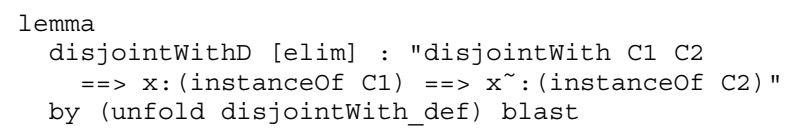

Therefore, the final proof is as follows.

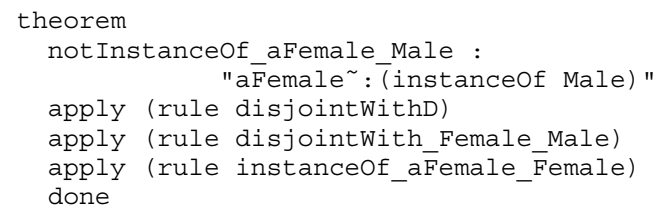

\subsection{Instance property reasoning}

Instance property reasoning is another important aspect of reasoning about Semantic Web ontologies. A Semantic Web application serves meaningful queries based on the understanding of ontology. Sometimes the necessary information is not directly stored in the database. The agent has to analyze what it knows to reply to the queries. We take a common relation between two persons as an example. Suppose a web resource Adam hasFather Peter. Can we conclude that Peter hasChild Adam? In order to answer this question, we have to define two more properties hasFather and haschild. Let hasFather be a subproperty of hasParent, and haschild be the inverse of hasparent.

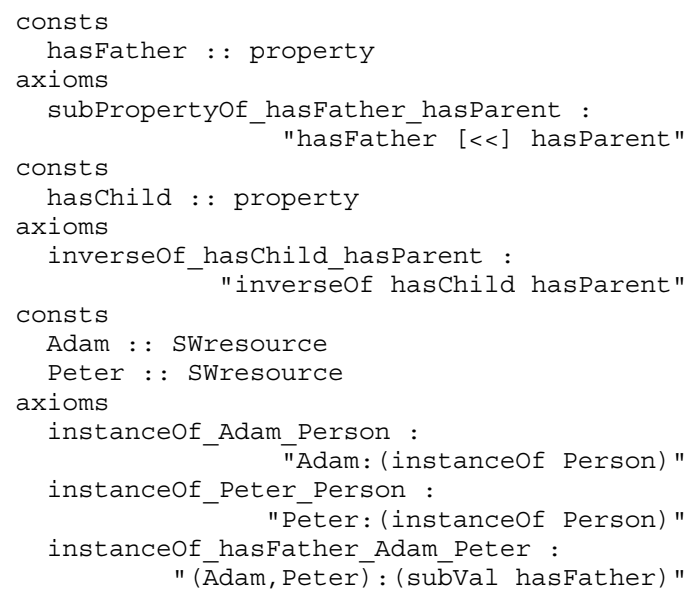

Two supporting lemmas are defined to help on the above query.

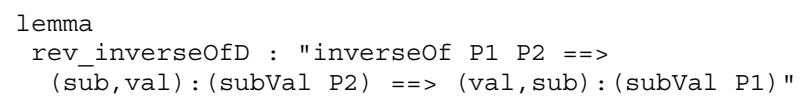




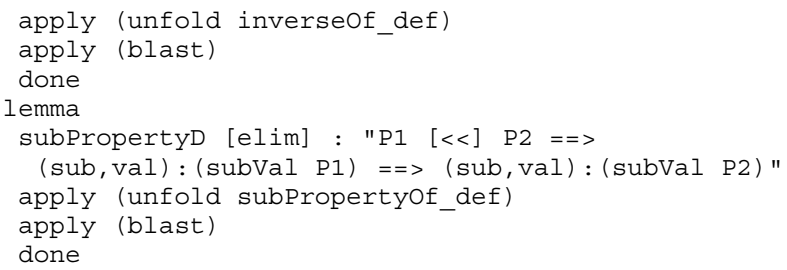

By applying these two lemmas and three available axioms, the goal to prove Peter haschild Adam can be reached step by step. Since hasFather is sub-property of hasParent, the fact Adam hasFather Peter ensures Adam hasParent Peter. Furthermore, haschild is inverse of has Parent. Now we can conclude that Peter hasChild Adam.

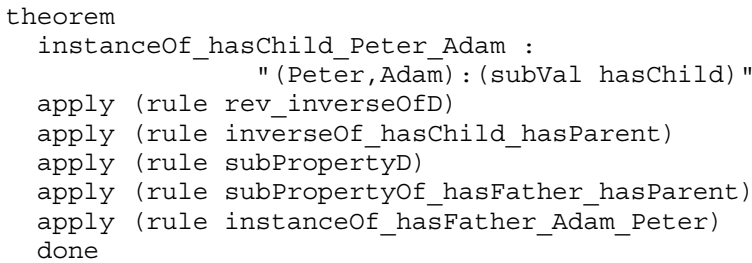

In this section, we discussed the different reasoning tasks performed by Isabelle through a 'human being' ontology example. we presented each reasoning proof together with the supporting lemmas used. In our implementation, we defined a sufficient set of supporting lemmas in the SW. thy theory library for assisting effective reasoning.

\section{Conclusion}

In this paper, we demonstrate an approach of reasoning about Semantic Web ontologies using generic theorem prover Isabelle. First, an Isabelle representation of the DAML+OIL semantic web meta-language was defined, which can be used as a theory library for detailed ontology modeling in Isabelle/HOL. Furthermore, a set of supporting lemmas were developed for the purpose of effective reasoning. Second, a DAML2Isa transform tool was developed for the automatic translation from DAML+OIL ontologies into their corresponding Isabelle theory files. Third, a case study illustrated the detailed procedures for reasoning about Semantic Web ontologies in Isabelle/HOL. Three types of reasoning tasks are discussed, i.e., subsumption, instantiation and instance property reasoning. In conclusion, Isabelle can check the consistency of Semantic Web ontology at both conceptual level and instance level. At the same time, it can help answer queries that are not included in the knowledge base. In summary, we believe that the generic theorem prover Isabelle can play a contribution role in the reasoning about Semantic Web ontologies.

One part of the future work is to enhance the general Isabelle theory library SW. thy. It will be more effective if the theory includes not only essential functions but also a sufficient set of supporting lemmas. Isabelle is a generic theorem prover. As a result, it lacks complete automation. Hence, another part of the future work is to reduce user interactions as much as possible so that the reasoning procedure can become more efficient. As discussed earlier, model checkers and theorem provers complement to each other. To improve the performance of reasoning about Semantic Web ontologies, it could be a better solution that Isabelle is worked together with a model checker such as Alloy.

\section{References}

[1] S. Bechhofer, I. Horrocks, C. Goble, and R. Stevens. OilEd: a reason-able ontology editor for the semantic web. In Proc. of the Joint German/Austrian Conf. on Artificial Intelligence (KI 2001), pages 396-408. Springer-Verlag, 2001.

[2] T. Berners-Lee, J. Hendler, and O. Lassila. The semantic web. Scientific American, May 2001

[3] J. Broekstra, M. Klein, S. Decker, D. Fensel, and I. Horrocks. Adding formal semantics to the web: building on top of RDF Schema. In ECDL Workshop on the Semantic Web: Models, Architectures and Management, 2000.

[4] R. S. Cost, T. Finin, A. Joshi, Y. Peng, C. Nicholas, I. Soboroff, H. Chen, L. Kagal, F. Perich, Y. Zou, and S. Tolia. Ittalks: A case study in the semantic web and daml.

[5] J. S. Dong, C. H. Lee, Y. F. Li, and H. Wang. Verifying DAML+OIL and beyone in Z/EVES. In The 26th International Conference on Software Engineering (ICSE'04), Scotland, UK, May 2004. IEEE Press.

[6] J. S. Dong, J. Sun, and H. Wang. Checking and Reasoning about Semantic Web through Alloy. In Proceedings of 12th Internation Symposium on Formal Methods Europe: FM'03, Pisa, Italy, Sept. 2003. LNCS, Springer-Verlag.

[7] R. Fikes and D. L. McGuinness. An axiomatic semantics for RDF, RDF Schema, and DAML+OIL. Technical Report KSL-01-01, Knowledge Systems Laboratory, 2001.

[8] V. Haarslev and R. Möller. RACER system description. In Proceedings of Automated Reasoning: First International Joint Conference, pages 701-706. Siena, June 2001.

[9] I. Horrocks. The FaCT system. Tableaux'98, Lecture Notes in Computer Science, 1397:307-312, 1998.

[10] I. Horrocks, U. Sattler, and S. Tobies. Practical reasoning for very expressive description logics. Logic Journal of the IGPL, 8(3):239-263, 2000.

[11] T. Nipkow, L. C. Paulson, and M. Wenzel. Isabelle/HOL A Proof Assistant for Higher-Order Logic, volume 2283 of LNCS. Springer, 2002.

[12] M. Saaltink. The Z/EVES system. In J. P. Bowen, M. G. Hinchey, and D. Till, editors, ZUM'97: Z Formal Specification Notation, volume 1212 of Lecture Notes in Computer Science, pages 72-85. Springer-Verlag, 1997.

[13] F. van Harmelen, P. F. Patel-Schneider, and I. H. (editors). Reference description of the DAML+OIL ontology markup language. Contributors: T. Berners-Lee, D. Brickley, D. Connolly, M. Dean, S. Decker, P. Hayes, J. Heflin, J. Hendler, O. Lassila, D. McGuinness, L. A. Stein, ..., March, 2001. 